\title{
SPECTROSCOPIC CONFIRMATION OF AN EXTREME STARBURST AT REDSHIFT $4.547^{1}$
}

\author{
Peter Capak ${ }^{2,3}$ C. L. Carilli,${ }^{4}$ N. Lee,${ }^{4}$ T. Aldcroft,${ }^{5}$ H. Aussel,${ }^{6}$ E. Schinnerer, ${ }^{7}$ G. W. Wilson, ${ }^{8}$ M. S. Yun ${ }^{8}$ \\ A. Blain, ${ }^{3}$ M. Giavalisco, ${ }^{8}$ O. Ilbert, ${ }^{9}$ J. Kartaltepe, ${ }^{9}$ K.-S. Lee,${ }^{10}$ H. McCracken, ${ }^{11}$ B. Mobasher,${ }^{12}$ \\ M. Salvato, ${ }^{3}$ S. Sasaki,${ }^{13}$ K. S. Scott,${ }^{8}$ K. Sheth,${ }^{2,3}$ Y. Shioya,${ }^{13}$ D. Thompson,${ }^{14}$ M. Elvis, ${ }^{5}$ \\ D. B. Sanders, ${ }^{9}$ N. Z. Scoville, ${ }^{3}$ and Y. Tanaguchi ${ }^{13}$ \\ Received 2008 April 4; accepted 2008 June 4; published 2008 June 27
}

\begin{abstract}
We report the spectroscopic confirmation of a submillimeter galaxy (SMG) at $z=4.547$ with an estimated $L_{\mathrm{IR}}$ $=(0.5-2.0) \times 10^{13} L_{\odot}$. The spectra, mid-IR, and X-ray properties indicate the bolometric luminosity is dominated by star formation at a rate of $>1000 M_{\odot} \mathrm{yr}^{-1}$. Multiple, spatially separated components are visible in the Ly $\alpha$ line with an observed velocity difference of up to $380 \mathrm{~km} \mathrm{~s}^{-1}$ and the object morphology indicates a merger. The bestfit spectral energy distribution and spectral line indicators suggest the object is 2-8 Myr old and contains $>10^{10} M_{\odot}$ of stellar mass. This object is a likely progenitor for the massive early-type systems seen at $z \sim 2$.
\end{abstract}

Subject headings: galaxies: evolution — galaxies: formation — galaxies: high-redshift galaxies: interactions — galaxies: starburst — submillimeter

\section{INTRODUCTION}

The study of galaxies detected at millimeter and submillimeter wavelengths is one of the most rapidly developing fields in observational astronomy. It is now known that a large fraction of the star formation activity is enshrouded in dust, with the star formation rate (SFR) being directly proportional to the far-infrared (FIR) luminosity of galaxies, modulo possible contributions from an active galactic nucleus (AGN) (Hughes et al. 1998). Surveys performed at millimeter wavelengths directly probe the FIR luminosity, and hence the amount of star formation. Furthermore, the shape of the galaxy spectral energy distributions (SEDs) at rest-frame millimeter wavelengths results in a negative $K$-correction in the range $0.5<z<10$. Therefore a flux-limited survey is equivalent to an SFR-limited survey at these redshifts (Blain et al. 2002).

The current redshift distribution of millimeter galaxies peaks at $z \sim 2$, with very few galaxies at $z>3$ (Chapman et al. 2005;

\footnotetext{
${ }^{1}$ Based on observations taken at the Keck Observatory, the James Clerk Maxwell Telescope, the Institut de Radioastronomie Millimetrique $30 \mathrm{~m}$ telescope, the Galaxy Evolution Explorer, the Chandra X-Ray Observatory, the Hubble Space Telescope, the Very Large Array, the Subaru Telescope, the United Kingdom Infrared Telescope, and the Canada-France-Hawaii Telescope.

${ }^{2}$ Spitzer Science Center, 314-6 Caltech, Pasadena, CA 91125.

${ }^{3}$ 105-24 Caltech, Pasadena, CA 91125.

${ }^{4}$ National Radio Astronomy Observatory, P.O. Box O, Socorro, NM 87801.

${ }^{5}$ Harvard-Smithsonian Center for Astrophysics, 60 Garden Street, Cambridge, MA 02138.

${ }^{6}$ AIM, Unité Mixte de Recherche CEA, CNRS, Université Paris VII, UMR 7158, Centre d'Études de Saclay, F-91191 Gif sur Yvette Cedex, France.

${ }^{7}$ Max-Planck-Institut für Astronomie, Königstuhl 17, Heidelberg D-69117, Germany.

${ }^{8}$ Astronomy Department, University of Massachusetts, Amherst, MA 01003.

${ }^{9}$ Institute for Astronomy, University of Hawaii, 2680 Woodlawn Drive, Honolulu, HI 96822.

${ }^{10}$ Department of Astronomy, 260 Whitney Avenue, Yale University, New Haven, CT 06511.

${ }^{11}$ Institut d'Astrophysique de Paris, UMR 7095 CNRS, Université Pierre et Marie Curie, 98 bis Boulevard Arago, 75014 Paris, France.

${ }^{12}$ Department of Physics and Astronomy, University of California, Riverside, CA 92521.

${ }^{13}$ Research Center for Space and Cosmic Evolution, Ehime University, 25 Bunkyo-cho, Matsuyama 790-8577, Japan.

${ }^{14}$ Large Binocular Telescope Observatory, University of Arizona, 933 North Cherry Avenue, Tucson, AZ 85721.
}

Pope et al. 2005; Aretxaga et al. 2007). However, the small bandwidth of current millimeter-wave spectrographs makes it very difficult to measure redshifts directly, and the low angular resolution of millimeter single-dish imaging leads to multiple optical candidates for the same source. As a result, millimeter surveys have relied on high-resolution radio data to identify the optical counterparts for subsequent spectroscopic follow-up. This leaves $35 \%-70 \%$ of the population of millimeter-selected galaxies at milli-Jansky flux levels unidentified and potentially at higher redshift (Wang et al. 2007; Younger et al. 2007).

In this Letter we report the discovery of a millimeter galaxy with a spectroscopic redshift of $z=4.547$ that appears to be dominated by star formation. This is the highest redshift galaxy detected at millimeter wavelengths not associated with an optically bright quasar. The object was independently selected as a Lyman break galaxy (K.-S. Lee et al. 2008, in preparation) for spectroscopic follow-up, a millimeter source, and a radio source (Carilli et al. 2008). The source reported here is unusual for millimeter sources because it has several nearby optically bright counterparts which were selected as $V$-band dropouts and is unusually luminous which allows for a radio detection. However, the confirmation of this object suggests the population of sources with similar radio to millimeter flux ratios and optical colors may also be at high redshifts.

We assume a $H_{0}=70, \Omega_{v}=0.7, \Omega_{m}=0.3$ cosmology and a star formation rate integrated across a Salpeter (1955) IMF from 0.1 to $100 M_{\odot}$ throughout this Letter.

\section{DATA}

Observations at $\lambda=1.1 \mathrm{~mm}$ with an average rms noise of 1.3 mJy were obtained with the AzTEC (Wilson et al. 2008) camera at the James Clerk Maxwell Telescope (JCMT) (Scott et al. 2008). Additional observations were obtained by the MAMBO camera on the IRAM $30 \mathrm{~m}$ telescope with an rms of $0.67 \mathrm{mJy}$ and a positional accuracy of $<5^{\prime \prime}$ (E. Schinnerer et al., in preparation). Ground-based optical and near-infrared imaging in 22 bands, Hubble Space Telescope, Spitzer, and Very Large Array images were obtained as part of the COSMOS survey as described in Capak et al. (2007), Scoville et al. (2007), Sanders et al. (2007), and Schinnerer et al. (2007), respectively. Additional deep $J$ and $K_{s}$ data were obtained with 
TABLE 1

OBJECT FLux

\begin{tabular}{|c|c|}
\hline Wavelength & $\begin{array}{l}\text { Flux }{ }^{\mathrm{a}} \\
(\mu \mathrm{Jy})\end{array}$ \\
\hline $0.2-8 \mathrm{keV}$ & $<3 \times 10^{-16} \mathrm{ergs} \mathrm{s}^{-1} \mathrm{~cm}^{-2}$ \\
\hline $150 \mathrm{~nm}(\mathrm{FUV})$ & $<0.2$ \\
\hline $250 \mathrm{~nm}$ (NUV) ...... & $<0.09$ \\
\hline $380 \mathrm{~nm}\left(u^{*}\right) \ldots \ldots \ldots$ & $<0.01$ \\
\hline $427 \mathrm{~nm} \ldots \ldots$ & $<0.02$ \\
\hline $446 \mathrm{~nm}\left(B_{\mathrm{J}}\right)$ & $<0.01$ \\
\hline $464 \mathrm{~nm} \ldots$ & $0.04 \pm 0.04$ \\
\hline $478 \mathrm{~nm}\left(g^{+}\right) \ldots \ldots \ldots$ & $0.03 \pm 0.02$ \\
\hline $484 \mathrm{~nm} \ldots \ldots \ldots \ldots$ & $0.04 \pm 0.04$ \\
\hline $505 \mathrm{~nm} \quad \ldots \ldots \ldots \ldots$ & $0.09 \pm 0.05$ \\
\hline $527 \mathrm{~nm} \ldots \ldots$ & $0.08 \pm 0.04$ \\
\hline $548 \mathrm{~nm}\left(V_{\mathrm{J}}\right) \ldots \ldots \ldots$ & $0.15 \pm 0.02$ \\
\hline $574 \mathrm{~nm} \ldots \ldots \ldots \ldots$ & $0.18 \pm 0.05$ \\
\hline $624 \mathrm{~nm} \ldots \ldots \ldots \ldots$ & $0.25 \pm 0.06$ \\
\hline $630 \mathrm{~nm}\left(r^{+}\right) \ldots \ldots \ldots$ & $0.50 \pm 0.03$ \\
\hline $679 \mathrm{~nm} \ldots \ldots \ldots \ldots$ & $1.24 \pm 0.06$ \\
\hline $709 \mathrm{~nm} \ldots \ldots \ldots \ldots$ & $1.30 \pm 0.07$ \\
\hline $711 \mathrm{~nm} \quad \ldots \ldots \ldots \ldots$ & $1.32 \pm 0.13$ \\
\hline $738 \mathrm{~nm} \ldots \ldots \ldots \ldots$ & $1.59 \pm 0.09$ \\
\hline $764 \mathrm{~nm}\left(i^{+}\right) \ldots \ldots \ldots$ & $1.60 \pm 0.05$ \\
\hline $767 \mathrm{~nm} \quad \ldots \ldots \ldots \ldots \ldots$ & $1.59 \pm 0.09$ \\
\hline $815 \mathrm{~nm} \ldots \ldots$ & $1.60 \pm 0.09$ \\
\hline $827 \mathrm{~nm}$ & $1.48 \pm 0.09$ \\
\hline $904 \mathrm{~nm}\left(z^{+}\right)$ & $1.82 \pm 0.10$ \\
\hline $1.25 \mu \mathrm{m}(J) \ldots \ldots \ldots$ & $2.4 \pm 0.8$ \\
\hline $2.15 \mu \mathrm{m}\left(K_{\mathrm{s}}\right) \ldots \ldots \ldots$ & $3.7 \pm 0.5$ \\
\hline $3.6 \mu \mathrm{m} \ldots \ldots \ldots \ldots$ & $7.9 \pm 0.2$ \\
\hline $4.5 \mu \mathrm{m} \ldots \ldots \ldots \ldots \ldots$ & $5.8 \pm 0.4$ \\
\hline $5.8 \mu \mathrm{m} \ldots \ldots \ldots \ldots \ldots$ & $3.4 \pm 1.3$ \\
\hline $8.0 \mu \mathrm{m} \ldots \ldots \ldots \ldots \ldots$ & $10 \pm 3.6$ \\
\hline $24 \mu \mathrm{m}$ & $26 \pm 13^{\mathrm{b}}$ \\
\hline $1.1 \mathrm{~mm}$ & $4800 \pm 1500$ \\
\hline $1.25 \mathrm{~mm} \ldots \ldots \ldots \ldots$ & $3410 \pm 670$ \\
\hline $20 \mathrm{~cm} \ldots \ldots \ldots \ldots \ldots$ & $45 \pm 9$ \\
\hline
\end{tabular}

${ }^{a}$ All limits are $1 \sigma$ limits.

${ }^{\mathrm{b}}$ A nearby bright source was modeled and subtracted to make this measurement; the formal upper limit is $70 \mu \mathrm{Jy}$ if the nearby source is not subtracted.

the UKIRT and CFHT telescopes as part of the COSMOS survey (H. McCracken et al., in preparation). X-ray data were obtained with the Chandra X-Ray Observatory as part of the C-COSMOS program (M. Elvis et al., in preparation). The respective fluxes are tabulated in Table 1.

The optical spectra were taken on the Keck II telescope using the DEIMOS instrument with a $1^{\prime \prime}$ slit width, the 830 line $\mathrm{mm}^{-1}$ grating (3.3 $\AA$ FWHM resolution), and the OG550 blocking filter to optimize red throughput. The data were collected in eight $1800 \mathrm{~s}$ exposures (4 hr total integration) under photometric conditions with $0.4^{\prime \prime}-0.6^{\prime \prime}$ seeing. The object was dith-

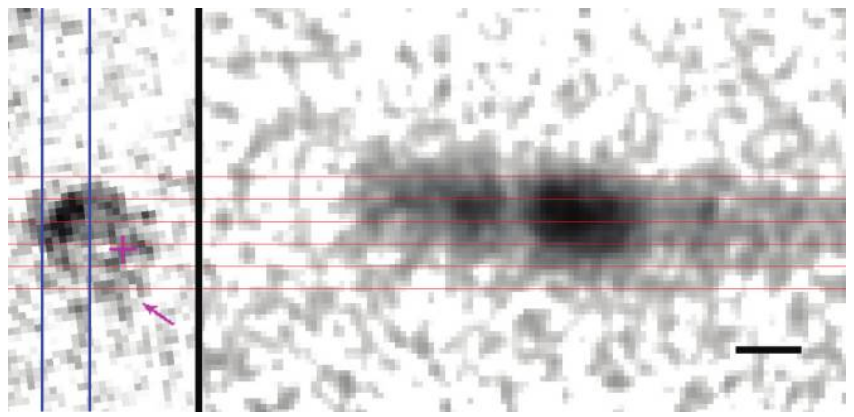

FIG. 2.-The 2D spectra of the object around the Ly $\alpha$ line is shown on the right along with the ground-based $z^{+}$-band image on the left with the slit position marked in blue, the radio position marked with a magenta cross, and the foreground object indicated by a magenta arrow. The red lines are spaced at $0.4^{\prime \prime}$ intervals, which is comparable to the spatial resolution of the spectra, and the black bar on the bottom right has a length of $1 \AA$ in the rest frame. Ly $\alpha$ emission is coming from all portions of the source and there is a clear velocity gradient along the slit. Note the Ly $\alpha$ absorption feature at the physical position of the bright continuum emission and the multiple peaks in the Ly $\alpha$ line.

ered along the slit by $\pm 3^{\prime \prime}$ between exposures to improve background subtraction. The data were reduced with a modified version of the DEEP2 DEIMOS pipeline (Marinoni et al. 2001). In addition to the standard processing this modified pipeline constructs and subtracts a median background and accounts for the shifts when combining the spectra. These additional steps remove the "ghosting" inherent to the 830 line $\mathrm{mm}^{-1}$ grating.

An image of the continuum and Ly $\alpha$ emission is shown in Figure 1 and indicates emission from all components of the source. The 2D and 1D spectra are shown in Figures 2 and 3, respectively. Ly $\alpha$ emission is detected from both the compact and extended portions of the object with a velocity gradient across the slit. Ly $\alpha$ emission from the diffuse region is redshifted with respect to the compact source, and a deep Ly $\alpha$ absorption feature is present at a redshift corresponding to other absorption features seen in the spectra. The dispersion of distinct peaks in the Ly $\alpha$ emission is $380 \mathrm{~km} \mathrm{~s}^{-1}$ and the line asymmetry indicates outflow winds of up to $\sim 1800 \mathrm{~km} \mathrm{~s}^{-1}$, typical of a merger with heavy star formation. Several interstellar absorption features are clearly seen in the continuum yielding a best-fit redshift of $4.547 \pm 0.002$, but the dispersion in the Ly $\alpha$ line suggests some components could be \pm 0.02 from the continuum redshift.

A foreground object with an $i^{+}$band flux of $0.58 \mu \mathrm{Jy}$ is visible to the west of our source in the $B$-band image (see Fig. 1) and its spectrum shows [O II] emission at $z=1.41$. The mass of this system is too low to significantly gravitationally lens our

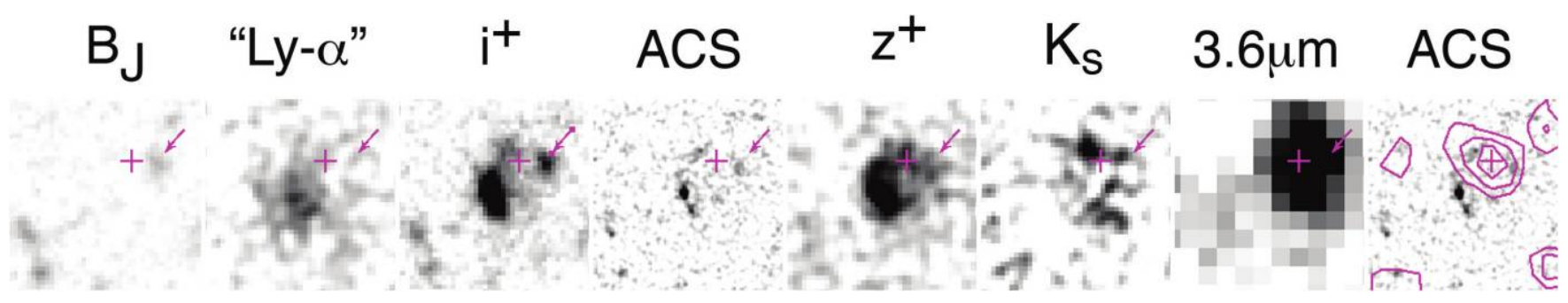

FIG. 1.-Images in $B_{\mathrm{J}}$, $\mathrm{Ly} \alpha, i^{+}$, ACS F814W, $z^{+}, K_{s}$, and $3.6 \mu \mathrm{m}$ are shown for $6^{\prime \prime} \times 6^{\prime \prime}(39.4 \mathrm{kpc})$ around the source. The radio position is marked with a cross and a foreground object is marked with an arrow. $1.4 \mathrm{GHz}$ Radio contours are overlaid on the ACS-band image in the rightmost panel. The Ly $\alpha$ image is generated by subtracting the $r^{+}$continuum image from the IA679 image which is centered on the Ly $\alpha$ line. The $3.6 \mu \mathrm{m}$ (rest-frame optical) flux is centered on the radio position and diffuse $i^{+}$and $z^{+}$(rest-frame UV) flux. A UV bright knot is visible down and to the left (0.6"to the SE) of the radio position and contains $73 \%$ of the UV flux. The Ly $\alpha$ emission is more extended than the UV emission seen in the $i^{+}$band and originates from both the UV bright knot and the radio position but not the foreground objects. Note that the UV bright knot is absent in the $K_{s}$-band image, indicating a very young age. 


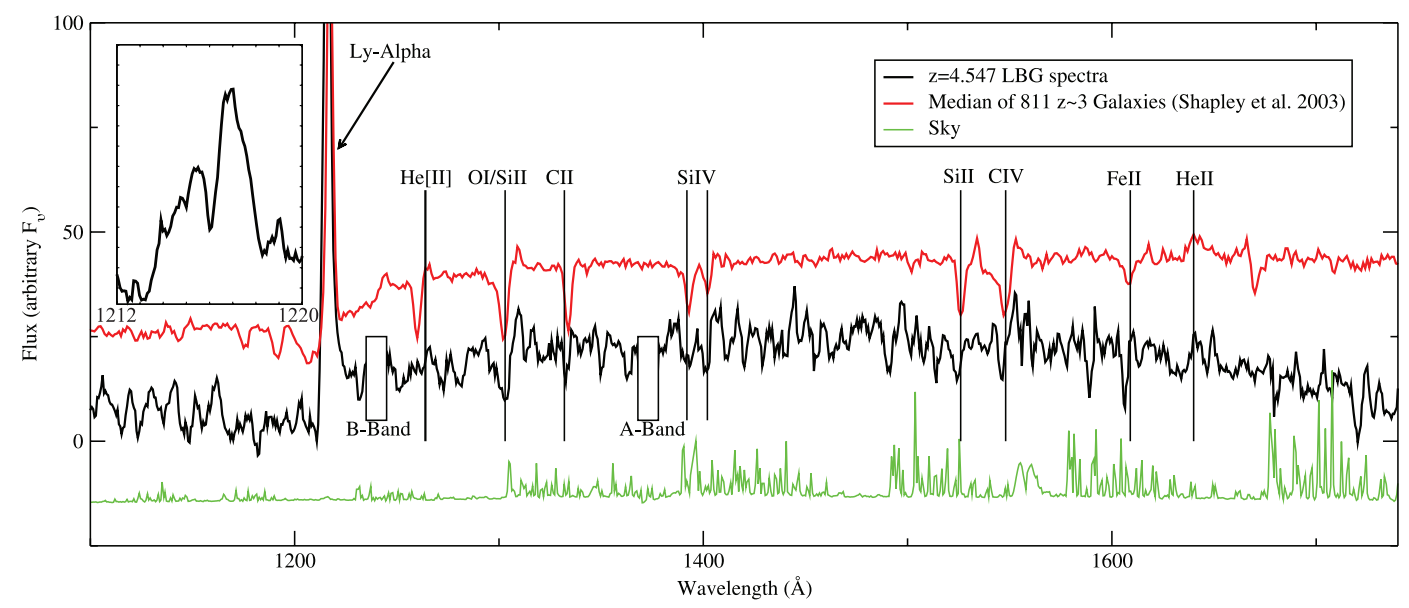

FIG. 3.-Optical spectrum of the source (black) is shown along with the composite LBG spectrum (red) from Shapley et al. (2003). The subtracted sky spectrum is shown in green for comparison and the atmospheric A and B absorption bands are marked with boxes. The inset panel on the right shows the region around the Ly $\alpha$ line. Lines from the interstellar medium and stellar photospheres such as O I, O II, C II, C IV, Si II, Fe II, and He II are clearly visible in the spectra. The presence of the Si IV $1297 \AA$ and C IV $1549 \AA$ P Cygni lines, along with the He II $1640 \AA$ emission lines, indicates the presences of both Wolf-Rayet and O stars, placing the age of the burst at a few Myr (Pettini et al. 2000). Note the absence of a common forbidden He [II] absorption feature at $1264 \AA$ which is generated in shocked gas, suggesting that we are not looking through a significant column of gas.

system and it is unlikely that the millimeter flux originates from this source because the object is outside the radio and Spitzer positional error and has an SED which indicates little obscuration. Furthermore, if the source of the millimeter emission is at $z=1.41$ the $24 \mu \mathrm{m}$, millimeter, and radio flux place the farinfrared luminosity at $\sim 10^{14} L_{\odot}$ and the dust temperature at less than $20 \mathrm{~K}$, which is physically unreasonable (Solomon \& Vanden Bout 2005; Dale \& Helou 2002; Carilli \& Yun 1999).

\section{PAN-CHROMATIC PROPERTIES AND MORPHOLOGY}

The rest-frame ultraviolet (UV) properties indicate a merger, typical of star-forming millimeter sources seen at $z \sim 2$ (Chapman et al. 2005; Pope et al. 2005; Aretxaga et al. 2007). At least two distinct components are visible in the ACS image, and a region of extended emission is visible in the groundbased $i^{+}$and $z^{+}$images, which are more sensitive to extended emission than the $H S T$ data. The rest-frame UV is centered at $10^{\mathrm{h}} 00^{\mathrm{m}} 54.516^{\mathrm{s}},+2^{\circ} 34^{\prime} 35.17^{\prime \prime}$ with the radio and rest-frame optical emission centered at $10^{\mathrm{h}} 00^{\mathrm{m}} 54.48^{\mathrm{s}},+2^{\circ} 34^{\prime} 35.9^{\prime \prime}$.

The majority $(73 \%)$ of the rest-frame UV flux originates from a compact $(<2.7 \mathrm{kpc})$ knot of emission at the southeast corner of the object, with the remaining UV emission extended over $17.7 \mathrm{kpc}\left(2.7^{\prime \prime}\right)$, while the rest-frame optical (Spitzer IRAC) flux of the source is centered on the diffuse UV and radio emission. The IA679 filter corresponds to the rest-frame Ly $\alpha$ line and shows strong emission from all components of the source and an extended Ly $\alpha$ halo around the source (see Fig. 1). In addition, a significant excess of flux is measured in the $3.6 \mu \mathrm{m}$ band which is centered on the $\mathrm{H} \alpha$ line at $z=$ 4.547. The Ly $\alpha$ image, the $3.6 \mu \mathrm{m}$ excess, and the optical-IR

TABLE 2

BEST-FIT SEDs

\begin{tabular}{ccccccc}
\hline \hline $\begin{array}{c}\text { Emission } \\
\text { Source }\end{array}$ & $\begin{array}{c}\text { Age } \\
(\mathrm{Myr})\end{array}$ & Average & $\begin{array}{c}\text { Mass } \\
\left(M_{\odot}\right)\end{array}$ & $\begin{array}{c}\text { UV SFR } \\
\left(M_{\odot}\right)\end{array}$ & $\chi^{2}$ & Model \\
\hline Diffuse $\ldots \ldots .$. & 6.5 & 1.4 & $1 \times 10^{10}$ & $3200 \pm 550$ & 24.3 & M05 \\
& 7.6 & 1.5 & $2 \times 10^{10}$ & $4200 \pm 730$ & 24.7 & BC03 \\
Compact $\ldots . . .$. & 2.5 & 0.0 & $2 \times 10^{8}$ & $250 \pm 20$ & 54.5 & M05 \\
& 2.9 & 0.0 & $6 \times 10^{8}$ & $250 \pm 20$ & 55.7 & BC03 \\
\hline
\end{tabular}

colors of the diffuse region are consistent with all components residing at $z=4.547$.

The possible presence of $\mathrm{H} \alpha$ in the $3.6 \mu \mathrm{m}$ band and [O II] in the $K_{s}$ band combined with the low $\mathrm{S} / \mathrm{N}$ of the $J$ band and multiple components with different SEDs make it difficult to constrain the age and mass of this object with stellar models because the $4000 \AA$ break strength is degenerate with the line ratio in some cases. To reduce these degeneracies $\mathrm{H} \alpha$ and [O II] line flux is added to the stellar models in proportion to the unobscured UV star formation rate (Kennicutt 1998). The diffuse portion of the source fades rapidly between the $K_{s}$ and $z^{+}$bands, indicating significant obscuration. The UV compact region is not detected redward of the $z^{+}$band, indicating a very young $(<5$ Myr old) stellar population and little dust obscuration.

A two-component fit to the total integrated light produced poor results. The UV and optical light are spatially separated, but the best-fit model places the majority of both the UV and optical light in a single unobscured $>0.1 \mathrm{Gyr}$ old population. To overcome this degeneracy we attempt to deconvolve the UV bright knot and the diffuse component of the source. Flux measurements were made in all of the ground-based images for the UV diffuse portion of the source using a 1 " diameter aperture on the original stacked images without PSF matching (Capak et al. 2007). An aperture correction of 1.4 mag, based on the ACS morphology, was then applied. A Maraston (2005, hereafter M05) or Bruzual \& Charlot (2003, hereafter BC03) single-burst model with solar metallicity, a Salpeter IMF, and a Calzetti et al. (2000) extinction law were then fit to these and the IRAC measurements. The model fluxes were then subtracted from the total flux, and a second model was fit to the remaining flux. The resulting fits are tabulated in Table 2 and shown in Figure 4.

The present data do not constrain the peak or shape of the FIR emission, so the total luminosity is uncertain, but the millimeter and $24 \mu \mathrm{m}$ data imply an infrared luminosity of $(0.5-2) \times 10^{13}$ $L_{\odot}$, and a corresponding SFR of $1000-4000 M_{\odot} \mathrm{yr}^{-1}$ (Dale \& Helou 2002; Chary \& Elbaz 2001; Carilli \& Yun 1999). The radio flux gives a second estimate of the SFR independent of dust obscuration at $3700 \pm 700 M_{\odot} \mathrm{yr}^{-1}$ assuming a radio spectral slope of -0.8 (Kennicutt 1998). Finally, assuming the excess flux in the $3.6 \mu \mathrm{m}$ band is due to $\mathrm{H} \alpha$ originating from the diffuse com- 


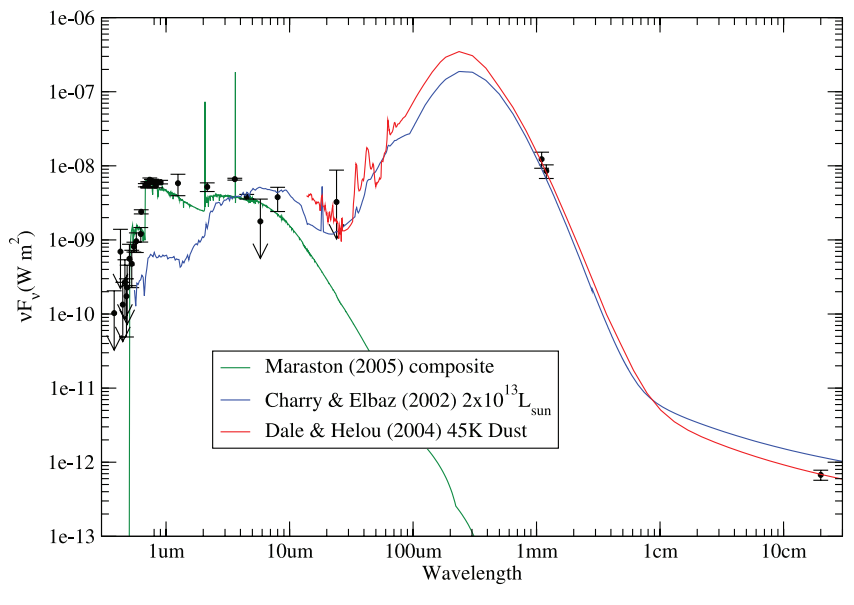

FIG. 4.-The pan-chromatic SED of the source is shown along with the best-fit SED model. The UV bright portion of the source is fit by a $2.5 \mathrm{Myr}$ old M05 stellar population with $1.9 \times 10^{8} M_{\odot}$ of stellar mass and no extinction, while the UV diffuse portion is fit by a $6.5 \mathrm{Myr}$ old population with $9.7 \times$ $10^{9} M_{\odot}$ of stellar mass and $1.4 \mathrm{mag}$ of visual extinction. The $8 \mu \mathrm{m}, 24 \mu \mathrm{m}$, millimeter, and radio portion of the SED is fit by a $2 \times 10^{13} L_{\odot}$ Chary \& Elbaz (2001) model (blue) or a $45 \mathrm{~K}$ Dale \& Helou (2002) model (red).

ponent, we derive a dust-corrected star formation rate of $2900 \pm$ $100 M_{\odot} \mathrm{yr}^{-1}$ (Calzetti et al. 2000; Kennicutt 1998), in good agreement with the UV, but lower than the millimeter and radio determined values. This last measurement is largely independent of the models because the $\mathrm{H} \alpha$ flux was fixed to the model star formation rate rather than fit as an extra parameter.

The bolometric luminosity of this source appears to be dominated by star formation. The UV and radio morphology suggest a star formation rate density of $15-50 M_{\odot} \mathrm{yr}^{-1} \mathrm{kpc}^{-2}$, within the range of locally observed starbursts (Solomon \& Vanden Bout 2005; Sanders \& Mirabel 1996). However, without a highresolution map of the millimeter emission and gas it is not possible to form a clear picture of how the star formation is distributed. No X-ray flux is detected in a $200 \mathrm{ks}$ Chandra exposure placing the X-ray-to-FIR luminosity ratio in the starformation-dominated regime (Alexander et al. 2003, 2005), and the radio-to-FIR flux ratio falls on the local starburst relation (Yun et al. 2001). However, the limit on the X-ray-to-radio luminosity ratio does not rule out an AGN. The SFR inferred by the diffuse UV and optical emission can explain the FIR emission if the two are spatially related. Finally, the Ly $\alpha$ line is narrow and AGN emission lines such as broad $\mathrm{C}$ IV or $\mathrm{N} \mathrm{V}$ are not observed in the optical spectra, so any AGNs must either be heavily obscured and/or outside the spectrograph slit.

\section{IMPLICATIONS FOR GALAXY FORMATION}

This object is a likely progenitor for the massive $\left(>10^{11} M_{\odot}\right)$, old ( $>2$ Gyr), early-type systems seen in large numbers at $z \sim 2$ (McGrath et al. 2007; Kong et al. 2006; Daddi et al. 2005; Cimatti et al. 2004). The morphology and spectral properties of the passive galaxies indicate they formed in a single burst at $z>4$ (Cimatti et al. 2008; Stockton et al. 2008; Daddi et al. 2005). However, the density of passive $z \sim 2$ systems is $\sim 10^{-4} \mathrm{Mpc}^{-3}$ (Kong et al. 2006; Daddi et al. 2005), which is too high to be explained by the previous millimeter source redshift distribution (Cimatti et al. 2008).

The discovery of this object and other recent studies suggest the fraction of $z>4$ millimeter sources may be higher than previously thought. An $850 \mu \mathrm{m}$ flux limited sample is equivalent to a star formation rate limited sample at $0.5<z<7.5$ and the $z \sim$ 2 objects must have formed by $z \sim 4$ in order to have sufficient time to evolve into passive systems. With this redshift range and a star formation duration (duty cycle) of $50 \mathrm{Myr}$, a surface density of 200 objects per square degree is required for these sources to be progenitors of the $z \sim 2$ passive galaxy population. Objects brighter than $4 \mathrm{mJy}$ at $850 \mu \mathrm{m}$ would have a sufficiently high SFR to form $>10^{11} M_{\odot}$ passive systems within $50 \mathrm{Myr}$ and the density of such sources is sufficient to form the $z \sim 2$ passive galaxies if $\sim 30 \%$ of them are at $z>4$ (Borys et al. 2003). This fraction is well within the range recent studies place at $z>4$ (Younger et al. 2007; Wang et al. 2006; Chapman et al. 2005).

Support for this work was provided by the Spitzer Science Center which is operated by the Jet Propulsion Laboratory (JPL), California Institute of Technology, under NASA contract 1407, NASA through contract 1278386 issued by the JPL, and NASA grant HST-GO-09822. C. C. thanks the Max-PlanckGesellschaft and the Humboldt-Stiftung for support through the Max-Planck-Forschungspreis.

\section{REFERENCES}

Alexander, D. M., et al. 2003, AJ, 125, 383 . 2005, ApJ, 632, 736

Aretxaga, I., et al. 2007, MNRAS, 379, 1571

Blain, A. W., et al. 2002, Phys. Rep., 369, 111

Borys, C., et al. 2003, MNRAS, 344, 385

Bruzual, G., \& Charlot, S. 2003, MNRAS, 344, 1000

Calzetti, D., et al. 2000, ApJ, 533, 682

Capak, P. L., et al. 2007, ApJS, 172, 99

Carilli, C. L., \& Yun, M. S. 1999, ApJ, 513, L13

Carilli, C. L., et al. 2008, ApJ, submitted

Chapman, S. C., et al. 2005, ApJ, 622, 772

Chary, R., \& Elbaz, D. 2001, ApJ, 556, 562

Cimatti, A., et al. 2004, Nature, 430, 184

2008, A\&A, 482, 21

Daddi, E., et al. 2005, ApJ, 626, 680

Dale, D. A., \& Helou, G. 2002, ApJ, 576, 159

Hughes, D. H., et al. 1998, Nature, 394, 241

Kennicutt, R. C., Jr. 1998, ARA\&A, 36, 189

Kong, X., et al. 2006, ApJ, 638, 72
Maraston, C. 2005, MNRAS, 362, 799 (M05)

Marinoni, C., et al. 2001, preprint (astro-ph/0109164)

McGrath, E. J., Stockton, A., \& Canalizo, G. 2007, ApJ, 669, 241

Pettini, M., et al. 2000, ApJ, 528, 96

Pope, A., et al. 2005, MNRAS, 358, 149

Salpeter, E. E. 1955, ApJ, 121, 161

Sanders, D. B., \& Mirabel, I. F. 1996, ARA\&A, 34, 749

Sanders, D. B., et al. 2007, ApJS, 172, 86

Schinnerer, E., et al. 2007, ApJS, 172, 46

Scott, K. S., et al. 2008, MNRAS, 385, 2225

Scoville, N., et al. 2007, ApJS, 172, 38

Shapley, A. E., et al. 2003, ApJ, 588, 65

Solomon, P. M., \& Vanden Bout, P. A. 2005, ARA\&A, 43, 677

Stockton, A., et al. 2008, ApJ, 672, 146

Wang, W.-H., Cowie, L. L., \& Barger, A. J. 2006, ApJ, 647, 74

Wang, W.-H., et al. 2007, ApJ, 670, L89

Wilson, G. W., et al. 2008, MNRAS, 386, 807

Younger, J. D., et al. 2007, ApJ, 671, 1531

Yun, M. S., Reddy, N. A., \& Condon, J. J. 2001, ApJ, 554, 803 